\title{
Challenge of handling a Charcot spinal arthropathy with a novel hybrid fibular autograft and expandable cage
}

\author{
Nissim Ohana, MD, ${ }^{1}$ Daniel Benharroch, MD, ${ }^{2}$ and Dimitri Sheinis, MD1 \\ ${ }^{1}$ Department of Orthopedic Surgery and ${ }^{2}$ Institute of Pathology, Soroka University Medical Center and Faculty of Health \\ Sciences, Ben Gurion University of the Negev, Beer Sheva, Israel
}

\begin{abstract}
A 26 -year-old man, who was paraplegic for 6 years due to a motor vehicle accident, presented to the authors' clinic following his incapacity to withstand a sitting posture, the frequent sensation of "clicks" in his back, and a complaint of back pain while in his wheelchair. On imaging, his dorsal spine showed a complete arthrodesis of the primarily fused vertebrae. However, distal to this segment, a Charcot spinal arthropathy with subluxation of T12-L1 was evident. Repair of this complex, uncommon, late complication of his paraplegia by the frequently used fusion techniques was shown to be inappropriate. A novel and elaborate surgical procedure is presented by which a complete fusion of the affected spine was secured. A left retrodiaphragmatic approach was used. Complete corpectomy of both the T-12 and L-1 vertebrae to the preserved endplates was performed. Most of the patient's fibula was resected and shaped for engrafting. The segment of the fibula was introduced into a mesh cage, before its intramedullary implantation into the T-12 and L- 1 vertebrae. This 2-step procedure combined the hybrid use of a fibular autograft and an expandable mesh cage, incorporated one into the other, in an innovative intramedullary position. This intervention allowed the patient to resume his former condition as an extremely physically active patient with paraplegia. Nine years later, an asymptomatic early-stage Charcot spine was found at L5-S1, but no treatment is planned at this point.
\end{abstract}

https://thejns.org/doi/abs/10.3171/2017.10.SPINE17606

KEYWORDS arthropathy; Charcot spine; cage; fibula; subluxation; surgical technique

$\sim_{1}$ HARCOT spinal arthropathy (CSA), also called neuropathic spinal joint disorder (Charcot spine), is a rare destructive medical condition secondary to a local innervation deficit, mainly resulting from a persistent paralysis of the limbs subsequent to traumatic damage to the spine. A pseudarthrosis follows distally to the fixed segment of the spine., ${ }^{4,12}$ The CSA is considered the late sequel of old traumatic damage progressing to a degenerative lesion, highlighted by a lack of proprioceptive sensation and occurring rarely in paralyzed and mainly in paraplegic patients. ${ }^{1}$

The diagnosis of Charcot spine is often delayed, in part due to an incomplete awareness of the early symptoms and signs. Among these are spinal distortion, loss of balance upon sitting, involvement of a long spine segment by fixation, excessive pressure on the spine, and a state after laminectomy; part or all the above should draw attention to a possible diagnosis of CSA. ${ }^{1}$

At presentation, this condition is heralded by difficulties in maintaining a sitting position, an impression of falling forward, ${ }^{5}$ the feeling of picking up recurrent "clicks," and evidence of an often painful protuberance over the old surgical scar on the back. Some surgeons have advocated a conservative treatment, based on the assumption that the hypermobility of the spine contributes to the independence of the patient with paraplegia. ${ }^{4,9}$ But treatment of the CSA is primarily surgical. The need for fixation is challenging, because it must address the excessive mobility in a part of the spine that has previously been stable. Circumferential fixation (arthrodesis) of the spine has been used extensively in such patients. A 4-rod lumbosacral fixation has been added by some surgeons.? 

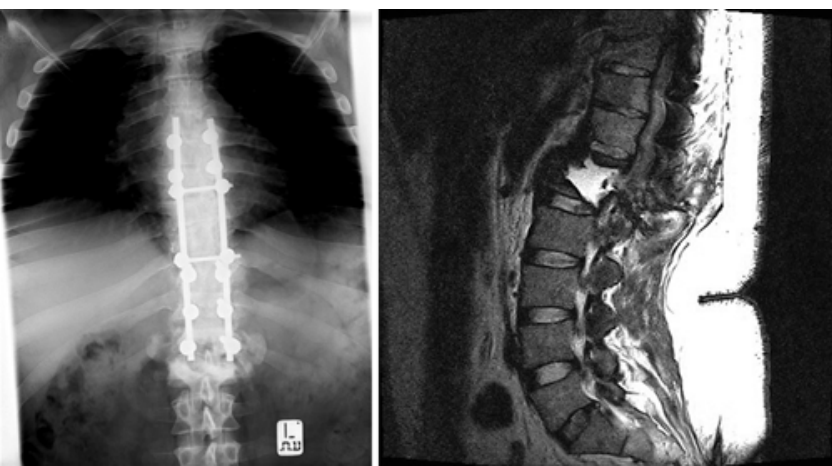

FIG. 1. Plain radiograph (left) and T2-weighted MR image (right) of the dorsal spine demonstrate a subluxation at T12-L1 consistent with Charcot spine distal to the previous arthrodesis. Note the fluid collection at the involved level.

An increasingly tighter circumferential fusion $\left(360^{\circ}\right)$, obtained by a combined anteroposterior access, limits the need for further revision procedures. A fibular allograft was introduced by Jacobs et al. into the increasingly complex interventions in an attempt to prevent a CSA relapse? These authors established the severe limitations of circumferential fusion in the surgical treatment of CSA.?

In this paper we report on a patient with Charcot spine who presented to our clinic with classic symptoms and signs. We highlight a novel procedure that had to be developed because of the failure of a common fusion technique used by our team in a preliminary operation. This combined surgical technique makes the best use of the patient's own fibula as well as of an expandable mesh cage to create the ultimate arthrodesis of the site affected by a prior excessive mobility.

\section{Case Report}

History and Examination

This 26-year-old man had been involved in a motor vehicle accident 6 years before his present admission. By then, he had a T8-9 fracture dislocation of the dorsal spine. He was operated on at another hospital, where he underwent T6-12 instrumented fusion (Universal Spinal System, DePuy Synthes). Following rehabilitation, he remained wheelchair-bound and despite his disability, he managed extremely well and was physically very active, using his hypertrophic upper limbs.

The patient presented at our clinic because of a painful bulging on his back, hearing frequent "clicks," and an impression of falling forward. On physical examination, he was bent forward, and a kyphosis was observed distal to the old scar on his upper back. His lower limbs were totally paralyzed.

On imaging, the complete arthrodesis of the fixed dorsal segment of the spine was preserved. Distal to this segment, a subluxation of the vertebrae was apparent (Fig. 1). A CT scan confirmed a T12-L1 subluxation with subtotal erosion of the L-1 vertebral body. An MR image revealed a fluid collection at the level of subluxation. No microorganisms were detected in a sample from the fluid (Fig. 1).

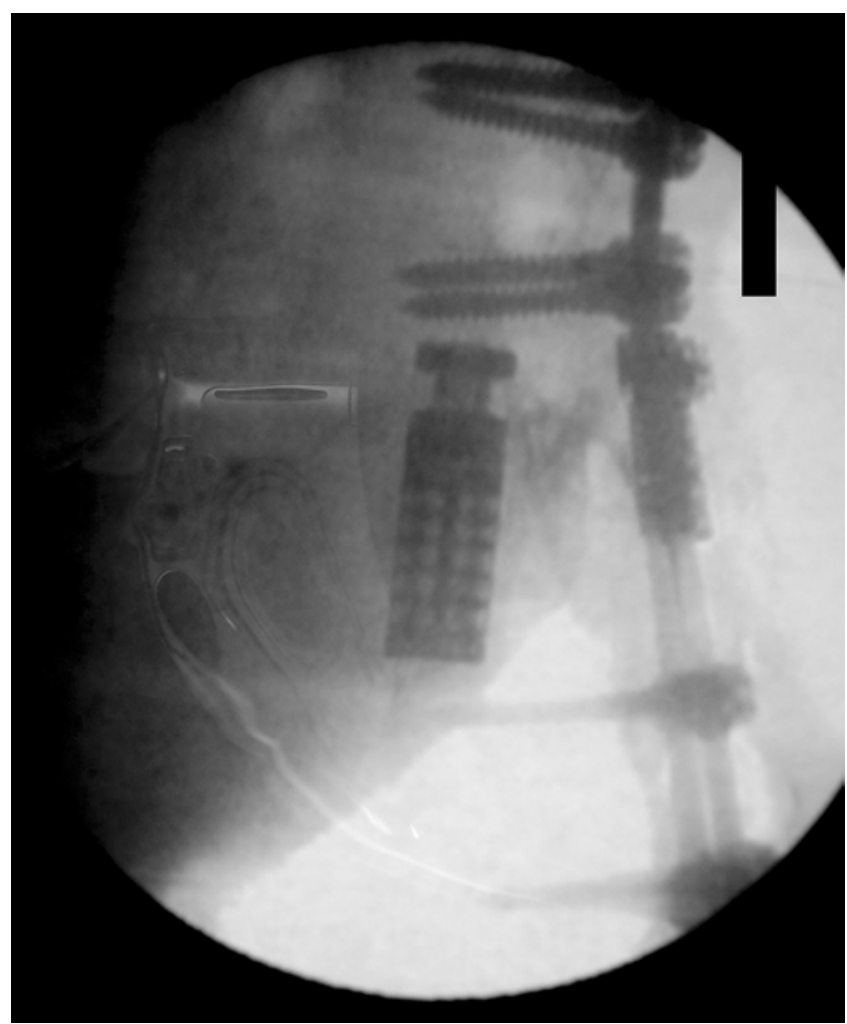

FIG. 2. Fluoroscopy of the thoracolumbar spine after the first surgery, with a mesh cage visible at the front.

\section{First Operation and Postoperative Course}

CSA was confirmed and surgery to fuse the affected spine was recommended. The first surgery consisted of removing the distal instrumentation of the previous fusion and removing the eroded fragments of the T-12 and L-1 vertebral bodies via a posterolateral approach. Subsequently, we reconstructed the spine with an expandable cage for anterior support and elongation of the instrumentation down to L-3 (Fig. 2).

The patient started a rehabilitation program. However, a few days later, he was observed leaning forward in a sustained manner. Complete breakup of the new fixation was confirmed on radiology, and the spine was declared unstable (Fig. 3), again. In addition, infection of the surgical wound was detected.

At this point, the patient's condition was complex: on one hand, no stable mechanical solution was readily foreseen. On the other hand, treatment of the wound infection was deemed urgent. The patient was taken to the operating room and the surgical wound was irrigated following debridement of the soft tissues. The posterior fixation was reestablished and consolidated to include the previously fixed segment of the spine, together with the distal spine down to L-5. In addition, beads of polymethylmethacrylate (PMMA), admixed with vancomycin powder, were placed in the anterior cavity formed in the spine at the site of the mesh cage to promote the treatment of the infection (Fig. 4).

The patient was then administered 6 weeks of intravenous antibiotics and confined to strict bed rest, except for 


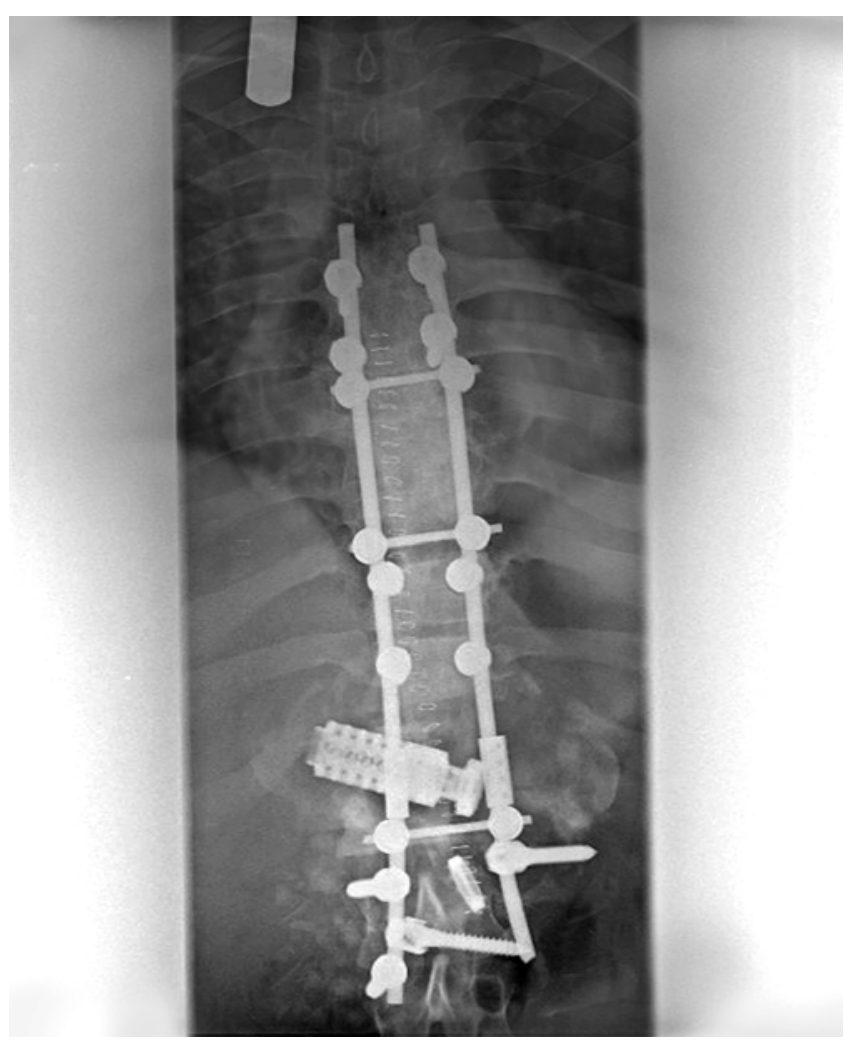

FIG. 3. Radiograph obtained shortly after our first surgery, demonstrating complete disruption of the instrumentation.

assisted postural changes. Only when the scar was healed and the markers of inflammation normalized was the next step performed.

\section{Second Operation and Postoperative Course}

In the operating room, the patient was positioned on his right side and a left thoracoabdominal incision was performed. The diaphragm, undamaged, was disconnected from its fixating tendons and the spine was exposed in a left retrodiaphragmatic approach. The damaged vertebrae were identified, and the PMMA beads were removed. Resection of the remnant T-12 and L-1 vertebral bodies was completed by corpectomy, down to the preserved end plates of the proximal and distal vertebrae. Next, the patient's left fibula was harvested in its central portion and was prepared for engrafting: it was measured, and then shaped at its proximal end to fit a tunnel prepared in the proximal vertebra and on its other end to adapt to the previously prepared funnel in the distal vertebra. Proximally, a tunnel was drilled using a flexible long-bone reamer. Distally, a slot was made along the vertebral body on its longitudinal axis. Before positioning the fibula, this bone was introduced into an expandable cage (Obelisc, Ulrich Germany) (Fig. 5) and the subsequent construct was employed to replace the missing part of the spine. When the construct was in place, the cage was expanded progressively until the acquisition of a complete support of its edges between the two endplates. Additional anterior fixation with rods and screws (Expedium, DePuy Synthes) was completed to

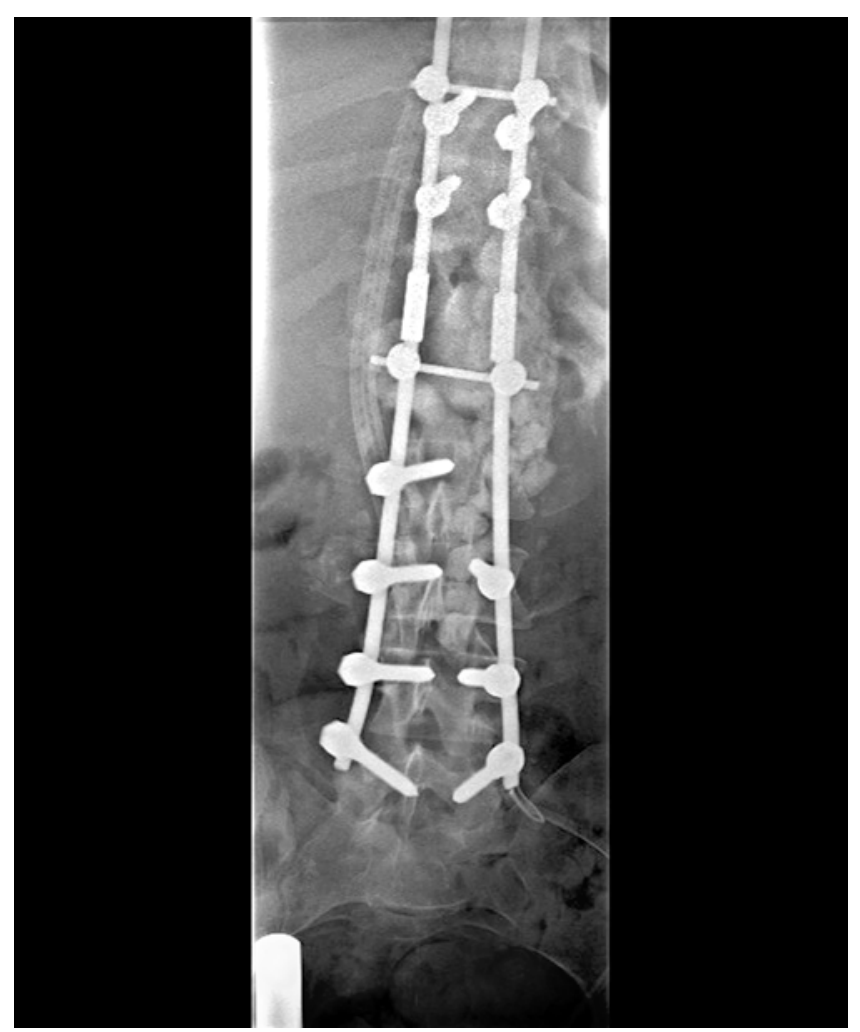

FIG. 4. Radiograph of the patient spine after the first step of our second surgery. The PMMA beads can be identified along the vertebral bodies.

encompass a solid fixation from T-10 to L-3, an addition to the posterior fixation from $\mathrm{T}-10$ to $\mathrm{L}-5$. Postoperative radiographs showed a stable fixation (Fig. 6). The unique characteristic of the construct was the intramedullary location of the fibular bone, in conjunction with an expandable cage, into which the bone was enclosed.

The patient was readmitted to the orthopedic ward. The drain was removed after a few days. Follow-up imaging showed a preserved fixed spine, including normal-looking grafts. The patient was confined to bed for another month, and then returned gradually to his former activity in the wheelchair. Serial follow-up radiology revealed a consistently adequate position of the grafts and incorporation of

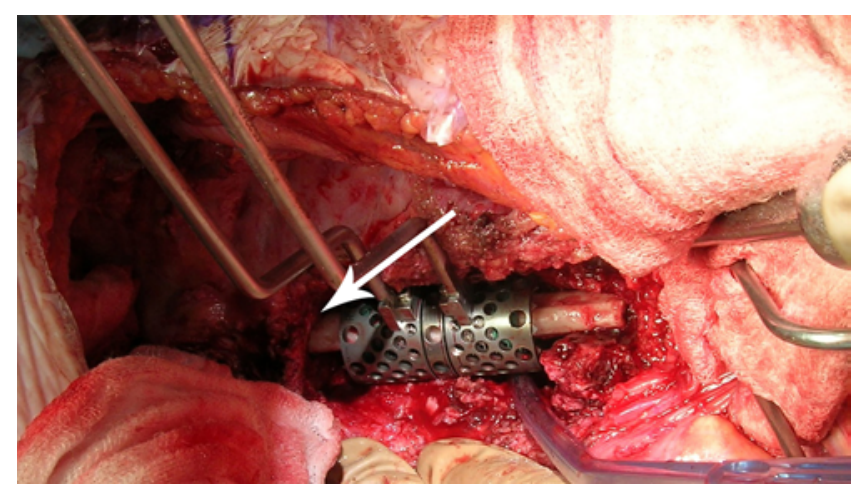

FIG. 5. Intraoperative view of the fibula-cage hybrid. The fibula is squeezed into the upper vertebral body (arrow). The cage is still in its preextension stage. 


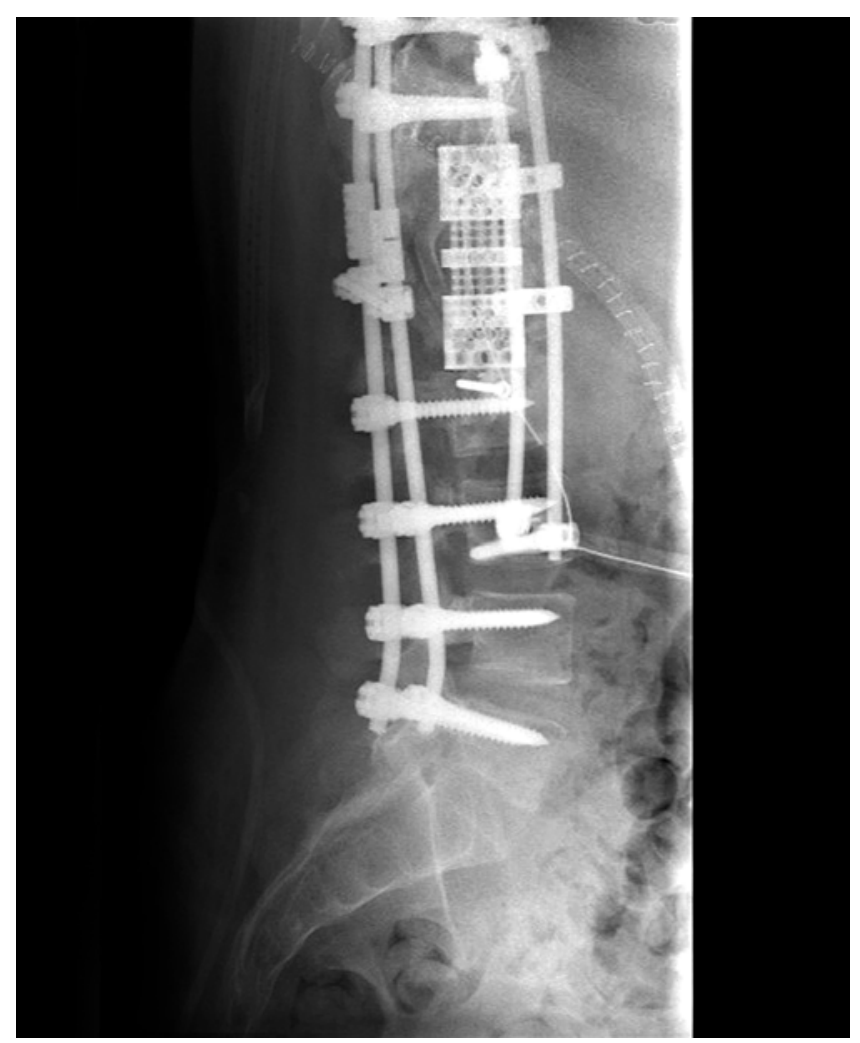

FIG. 6. Final postoperative radiograph of the patient's spine. The instrumentation alignment is well preserved.

the fibular segment to the spine, without any damage to the construct.

Six months after surgery, the patient was able to indulge in physical activity with or without the help of the wheelchair. Today, 9 years later, a complete arthrodesis is evident, with resumption of the patient's former functioning in and out of the wheelchair. The patient was lost to followup for about 3 years and he reappeared 9 years after our first surgery. He had completely regained his previous level of fitness, with no complaints. Evaluation of the fixation by CT scan showed complete arthrodesis, with excellent stability. However, the imaging revealed early evidence of a secondary Charcot spine at L5-S1, which was totally asymptomatic (Fig. 7).

\section{Discussion}

The patient reported in this paper demonstrates the classic features described as CSA, a degenerative spinal condition of incompletely clarified pathogenesis. ${ }^{17,13}$ Charcot spine diagnosis may be challenging, as the differential diagnosis includes discitis-osteomyelitis, tuberculosis, and spondyloarthropathy developing in the patient on hemodialysis. ${ }^{8}$ The first round of surgery was performed at another hospital and one wonders if the diagnosis of CSA was established at that stage.

However, we have elected to illustrate the unusual surgical procedures adopted as this rare condition evolved. Other orthopedic surgeons may have preferred circumferential fusion of the spine. ${ }^{7,10,12}$ But the surgical interven-

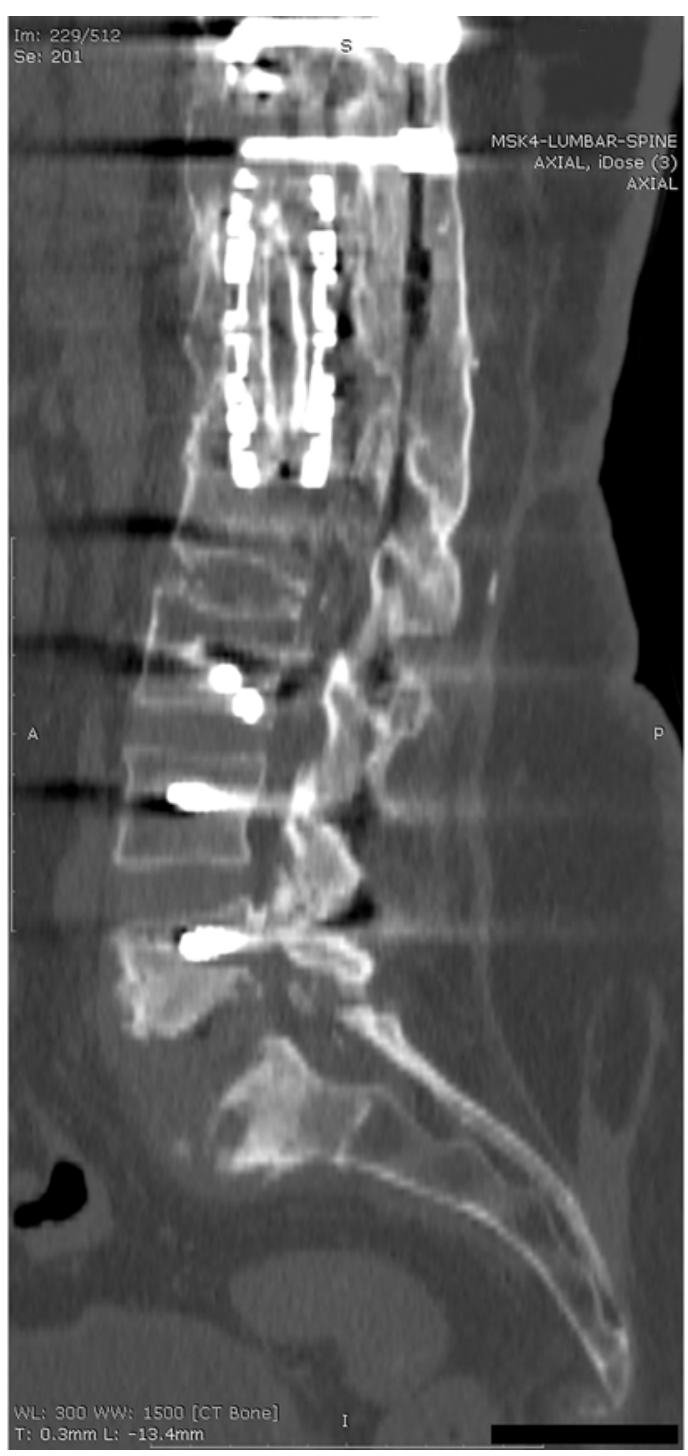

FIG. 7. Midsagittal CT reconstruction of the thoracolumbar spine, demonstrating a solid arthrodesis across the involved vertebrae. The fibula is identified within the cage in the vertebral bodies.

tion decided upon for our patient seemed to be the most adapted to the circumstances.

The use of fibular grafts in spinal surgery in different setups should be noted. In advanced spondylolisthesis conditions, fixation of the L5-S1 vertebrae is performed, using the Bohlman technique. Fibular allografts from a bone bank are introduced from behind through the S-2 into the L-5 vertebrae. ${ }^{11}$ However, the aim of this procedure is different from that described in our report, as the patients undergoing the Bohlman procedure show no pathological mobility of their vertebral column. Another use of fibular allografts is for anterior fixation of the cervical or dorsal spine. In this procedure, defects generated at surgery, such as those following the removal of a vertebra or a disc, are filled with fragments of fibula from a bank. In these cases the fibula serves mainly as a filling bone material. ${ }^{3}$

In contrast, our case may represent the first in which a significant fragment of fibula was positioned in an in- 
tramedullary location for the treatment of Charcot spine. This solution was adopted when it became evident that a complete mixed athrodesis may probably be the only effective treatment for our patient. It is not unlikely that this arthrodesis, with fixation of a long segment of spine, was the only outlet for a patient who is extremely active and alert when using his upper limbs. In fact, this excessive physical fitness may have been the origin of the Charcot spine. A more limited, fixed, spinal segment might have represented only a temporary solution. On one hand, fusion of the spine to the pelvis may have prevented the development of a secondary CSA in L5-S1. On the other hand, at that point in time, we were determined to preserve the patient's excellent physical condition, in spite of his invalidism. His athletic shape might have been at the origin of the primary CSA, as well as of the incipient secondary one. A high proportion of the patients in the series of Jacobs et al. " were "self-transferring patients" (able to transfer themselves from the wheelchair to bed), highlighting a high degree of fitness, as was our patient.

Because an excellent bone fusion was imperative, a fibula of autogenous origin was chosen. The composite use of a fibula and an expandable mesh cage was meant to multiply the maintenance system to the spine, as the cage supplied a temporary mechanical support until the incorporation of the fibular graft into the spine, which acted as a biological support. Although a fibular allograft had also been considered, we were not reluctant to sacrifice the patient's own fibula, as it was not of use in his case, while failure of an allograft might have jeopardized the entire construct. No additional materials were employed to strengthen the construct.

Although we are not aware of a comparison between a fibular allograft and a fibular autograft, a comparison involving a free vascularized fibula (itself an autograft) has been described. The latter was performed in cases of malignant tumor resections in the spine or pelvis, which required filling of the subsequent lacuna ${ }^{6}$ The free vascularized fibula showed superior results to the fibular allograft, reflecting our own results. The authors highlighted the increased solidity obtained with the free vascular fibula ${ }^{6}$

The combined use of fibular bone and a mesh cage for a reconstructive intervertebral graft has been described previously. ${ }^{6,14}$ The originality of our contribution lay in the intramedullary localization of the construct. The hybrid construct, including the autograft, was not meant to impart additional strength or rigidity. For that purpose, we had installed the cage and an anterior screw-rod construct from T-10 to L-3, and a posterior fixation from T-10 to L-5, which provided an expected solidity to the spine. Our concept is based on the following thinking: in the neuropathic spine, as compared with a nonneuropathic one, we are pressed for time, so the fusion may progress and be finalized. The regular patient is at first immobilized by pain, then as healing occurs, he will gradually return to his previous level of activity. This is not the case for the neuropathic patient who, due to an absence of pain perception, has no restriction in movements, but shows also a deficient inflammatory reaction and repair. A metallic construct in the spine of such a patient is therefore more brittle, and may give way to a pseudarthrosis, as was the case in one of the first stages with our patient. The fibular graft was suggested to restrict free-floating movement within the spine. Moreover, the fibula was expected to accelerate the process of fusion, and therefore to consolidate the merging of the construct in the long term. But a biomechanical analysis was not performed in this case.

One may infer from this report that when the diagnosis of CSA is made, the surgeon should initiate an aggressive modality of treatment from the very first stage. Unfortunately, an extensive fixation does not necessarily preclude the development of a secondary Charcot spine. Postoperative sequelae have encompassed implant loosening, development of Charcot disease in further joints, and wound infection. ${ }^{1}$

We believe the 9-year follow-up justifies the surgical procedure used for this patient. Imaging confirmed the extensive unification of the spine, notably at the level of the originally damaged vertebra. The absence of a large segment of the fibula is of no functional significance, especially in the patient with paraplegia. ${ }^{2}$ This type of intervention, although rendered necessary because of a very specific medical problem, may eventually be used again in further complex situations, including for CSA.

As mentioned above, this patient is remarkable for his high level of physical activity, which may be equivalent to that of an athlete. The damage to the spine over time, especially in the presence of a locally abnormal sensory system, might prevent any repair in the relevant vertebrae. Unfortunately, numerous young people suffer from paraplegia. Some of them may develop the stamina necessary for a high degree of activity. Nevertheless, CSA is a rare complication. This fact, together with the difficulty in diagnosing Charcot spine, especially at the incipient stages, has lead us to suggest the presence of a possible underdiagnosis of this condition.

\section{Acknowledgments}

We thank Kibbutz Sde-Boker for their support.

\section{References}

1. Aebli N, Pötzel T, Krebs J: Characteristics and surgical management of neuropathic (Charcot) spinal arthropathy after spinal cord injury. Spine J 14:884-891, 2014

2. Bozkurt M, Yavuzer G, Tönük E, Kentel B: Dynamic function of the fibula. Gait analysis evaluation of three different parts of the shank after fibulectomy: proximal, middle and distal. Arch Orthop Trauma Surg 125:713-720, 2005

3. Brazenor GA: Comparison of multisegment anterior cervical fixation using bone strut graft versus a titanium rod and buttress prosthesis: analysis of outcome with long-term followup and interview by independent physician. Spine (Phila Pa 1976) 32:63-71, 2007

4. Cleveland M, Wilson HJ Jr: Charcot disease of the spine; a report of two cases treated by spine fusion. J Bone Joint Surg Am 41-A:336-340, 1959

5. De Iure F, Chehrassan M, Cappuccio M, Amendola L: Sitting imbalance cause and consequence of post-traumatic Charcot spine in paraplegic patients. Eur Spine J 23 (23 Suppl 6):604-609, 2014

6. Houdek MT, Rose PS, Bakri K, Wagner ER, Yaszemski MJ, Sim FH, et al: Complications of reconstruction with use of free vascularized fibular graft for spinal and pelvic defects 
following resection of a malignant tumor. J Bone Joint Surg Am 99:e69, 2017

7. Jacobs WB, Bransford RJ, Bellabarba C, Chapman JR: Surgical management of Charcot spinal arthropathy: a single-center retrospective series highlighting the evolution of management. J Neurosurg Spine (Phila Pa 1976) 17:422-431, 2012

8. Ledbetter LN, Salzman KL, Sanders RK, Shah LM: Spinal neuroarthropathy: pathophysiology, clinical and imaging features, and differential diagnosis. Radiographics 36:783-799, 2016

9. Moreau S, Lonjon G, Jameson R, Judet T, Garreau de Loubresse C: Do all Charcot spine require surgery? Orthop Traumatol Surg Res 100:779-784, 2014

10. Proietti L, Pola E, Nasto LA, Scaramuzzo L, Logroscino CA: Onset of a Charcot spinal arthropathy at a level lacking surgical arthrodesis in a paraplegic patient with traumatic cord injury. Eur Spine J 19 (Suppl 2):S83-S86, 2010

11. Roca J, Ubierna MT, Cáceres E, Iborra M: One-stage decompression and posterolateral and interbody fusion for severe spondylolisthesis. An analysis of 14 patients. Spine (Phila Pa 1976) 24:709-714, 1999

12. Vialle R, Mary P, Tassin JL, Parker F, Guillaumat M: Charcot's disease of the spine: diagnosis and treatment. Spine (Phila Pa 1976) 30:E315-E322, 2005

13. Wirth CR, Jacobs RL, Rolander SD: Neuropathic spinal arthropathy. A review of the Charcot spine. Spine (Phila Pa 1976) 5:558-567, 1980
14. Yanamadala V, Rozman PA, Kumar JI, Schwab JH, Lee SG, Hornicek FJ, et al: Vascularized fibular strut autografts in spinal reconstruction after resection of vertebral chordoma or chondrosarcoma: A retrospective series. Neurosurgery 81:156-164, 2017

\section{Disclosures}

The authors report no conflict of interest concerning the materials or methods used in this study or the findings specified in this paper.

\section{Author Contributions}

Conception and design: Ohana. Acquisition of data: Ohana, Sheinis. Analysis and interpretation of data: Ohana. Drafting the article: Benharroch, Ohana. Critically revising the article: Benharroch, Sheinis. Approved the final version of the manuscript on behalf of all authors: Benharroch. Administrative/technical/material support: Ohana. Study supervision: Ohana.

\section{Correspondence}

Daniel Benharroch: Institute of Pathology, Soroka University Medical Center, Beer Sheva, Israel. danielbenharroch1@gmail. com. 